\title{
Management of Magnetic Resonance Imaging Waiting Lists
}

\author{
Christopher B. Lightfoot*, Margaret Hsieh
}

*Dalhousie Medical School Class of 2005

Abstract: Management of MRI waiting lists in Canada is inadequate. There is very little medical literature addressing this important topic. Increased capital investment by government might not be adequate to reduce wait times. We sought the impact of a local private MRI clinic on the region's public health care wait times. Wait times surprisingly increased following the opening of this private MRI clinic and did not significantly decrease over the next six months. However, this analysis was complicated by the closure of one of the public MRI sites and the use of an MRI machine at a paediatric tertiary care centre for some adult patients. Increased resources directed specifically towards the implementation of standards and accountability as well as capital and infrastructure investment might improve current and future delivery of diagnostic imaging.

$\mathrm{W}$ aiting lists have been described as an unavoidable part of publicly-funded health care systems. Waiting for medical service may, perhaps surprisingly, have some benefits. These could include permitting time for patients to reflect upon upcoming procedures, in particular when significant risk exists, as well as allowing for more conservative treatment choices during the waiting period. ${ }^{1}$ However, others suggest the more widely accepted stance that addressing and managing waiting lists is paramount because of concerns about extended suffering, disability and possible deaths that result from delays in provision of service. ${ }^{2}$

The current status quo in waiting list management for a variety of services in Canada is dismal and the management of magnetic resonance imaging (MRI) waiting lists is no exception. ${ }^{1-4}$ McDonald et al. surveyed Canadian hospitals with more than 100 acute care beds and found that only 60 per cent had systematic criteria in place for structuring MRI waiting lists. ${ }^{1}$ Furthermore, half of the respondents indicated that MRI waiting lists were usually or always a problem.

Unfortunately, further objective studies published on the topic of MRI waiting times are relatively scarce. It is common knowledge, however, that waiting lists are long and little is being done to assess the prioritisation of patients based on medical need. In the public system, where all individuals are treated equally, one might expect that patients are triaged on a greatest-need-for-intervention basis, yet this is a complex issue when dealing with diagnostic imaging. When compared with a surgical waiting list that has a well-defined intervention with easily defined outcomes, diagnostic imaging frequently evaluates non-treatable disease and is often only one of multiple interventions on the road to a given outcome. Therefore, when evaluating waiting lists, the likelihood that critical diagnostic information and subsequent treatment will improve outcomes must be taken into account. ${ }^{3}$

One well-established effort that attempted to address these issues was the Western Canada Waiting List Project, a federally funded partnership of 19 organisations created to develop tools for waiting list management. A pointscore tool was produced to categorise medical urgency for MRI scanning. This effort was the only one of its kind found through a Medline search (August, 2003). Reliabil- ity of the tool was assessed and the researchers found poor inter-rater reliability, but good test-retest reliability. ${ }^{2,3}$ It is clear that more effort needs to be made in this area.

There has been much debate recently on the role of private service in the Canadian health care system and the diagnostic imaging industry is no exception. Consider US health care as an example of a privatised system and Canadian health care being predominantly public. Bell et al. ${ }^{5}$ surveyed major hospitals in the US and Canada and found that MRI imaging of the head had waiting times of 3 and 150 days, respectively. Thus, assuming that many other factors are equal, a private system would appear to have shorter wait times. However, in the United Kingdom, where physicians operate in both private and public sectors, greater access to private care appears to have resulted in some longer public sector wait times. Audits have shown large numbers of patients experiencing waiting times that are likely to be inappropriate for their circumstances. ${ }^{1}$

One consideration in moving to a partially privatised diagnostic imaging system is to be able to ensure stable delivery of a service that is maintained on a "for profit" basis. Furthermore, the quality of the imaging information may be questioned, as an increase in imaging means more demand on physicians' time, which may subsequently result in slower access to specialists at the time of data acquisition. ${ }^{6}$ Roy Romanow's recent report "Building on values: the future of health care in Canada" has strongly criticised the role of the private imaging industry. He wrote, "it is eroding the equality of access" tenet of the Canada Health Act, believing people are being fast-tracked with private service and then jumping back into the public system further ahead than those who did not use private services. ${ }^{7}$ Romanow recommended that diagnostic services be explicitly classified under "insured health services", which currently includes "hospital and physician care" in order to clarify this service's role under the Canada Health Act, and thus remove it from the private sector. ${ }^{7,8}$ There has been suggestion in the media that physicians in some provinces are referring patients to private clinics with documentation that the tests are "medically not necessary" to avoid potential medico-legal repercussions. ${ }^{9}$ The confusing logistical aspects of uniting public and private service should indeed be clarified. 


\section{MRI in Nova Scotia}

In the fall of 2002, Nova Scotia became the fifth province with a private MRI clinic, located in Halifax. ${ }^{10}$ Prior to that time, the QEII Health Sciences Centre in Halifax had two MRI machines performing all the adult MRI imaging in the province. One of the MRI machines at the QEII was shut down for upgrading the same month the private MRI opened (Figure 1). In the months that followed the opening of the private MRI clinic, the IWK Health Centre in Halifax started performing selected MRI procedures on the adult population to assist in reducing wait times.

The primary goal of this analysis was to investigate the impact of the new private MRI clinic on the QEII MRI waiting list. We studied waiting times rather than

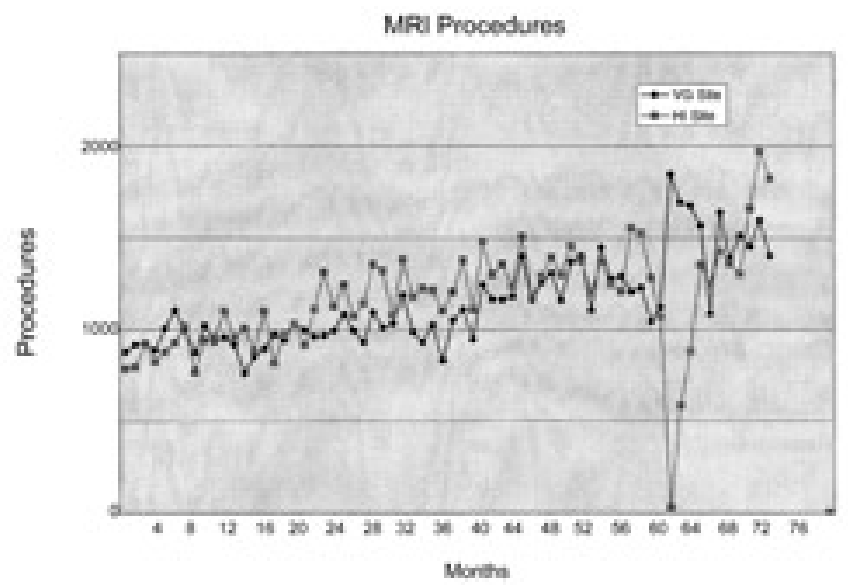

Figure 1: Numbers of MRI procedures over time.

volume of patients awaiting procedures because wait times may be more relevant. ${ }^{4}$ For example, a patient could be second on an organ transplant list and still wait years for the procedure, while 1000 patients listed for an endoscopy may be scoped in the same period of time. We acknowledge that accurate assessment of waiting times may be impossible. There may be inflation of up to 30 percent due to patient death, declining of procedures, or missing of appointments. ${ }^{1}$

\section{Methods}

A telephone interview was conducted in April 2003 wherein the primary investigator asked questions of the private MRI clinic staff. MRI waiting list data was collected from the QEII Health Sciences Centre Department of Radiology in April of 2003. The waiting lists prior to and following the introduction of the private MRI clinic were compared with a Student's t-test. As there were three months with below average numbers of procedures on one of the QEII MRI machines (Figure 1), these months were excluded from analyses. Trends in wait times were analysed with regression analyses. The paired, two-tailed Student's t-test was also used to evaluate the trend postintroduction of private MRI.

\section{Results}

\section{Information from private MRI clinic}

The following are the questions and answers from the telephone interview with the private MRI clinic:

\section{How much does it cost to have an MRI scan?}

The average rate is $\$ 725$ for a routine scan. The costs, of course, increase with various procedures such as gadolinium enhancement scans.

2. How many scans do you perform on an average day?

We perform approximately 8 per day. We are hoping to increase this number. We are working with two technologists at the moment. We would like to be up to $18-20$ scans per day. Since our inception, we have averaged approximately 125 per month.

3. How long does it take to get in and have a scan done?

There are currently appointments available for next week.

4. Who reads your scans and how do the patients find out results?

We have radiologists that come out in the evening to read the scans. The reports are generally done within $5-7$ days and the results are sent back to the referring physician who may be a specialist or a family physician.

\section{Who makes up your clientele?}

The majority are from Workers Compensation Board, insurance companies and just the public in general.

6. How does someone get in to see you?

We require a referral from a physician, chiropractor or other medical practitioner.

\section{Analysis of public MRI waiting lists}

Waiting times for months prior to and following the introduction of the private MRI clinic were graphed (Figure 2). Interestingly, overall waiting lists for public MRI procedures significantly increased following the opening of the private MRI clinic (Figure 3). Waiting times increased for angiography, body, bone, and neuro imaging. The only exception was the wait time for cardiac MRI, which had no significant change (Table 1).

The rate of change in waiting times was assessed (Figure 4). Regression analysis of the rate of change in waiting list over the months leading up to the opening of the MRI clinic resulted in a regression equation of $y=4.23 x+93.96$, while $y=-3.57 x+177.67$ was determined for the post-introductory period. Although there has been a downward trend in waiting times since the opening of the private MRI clinic, the change has not been significant ( $\mathrm{t} 4=2.45, \mathrm{P}=0.07$ ). Furthermore, the QEII scanners have steadily increased the number of procedures run per month over the past 63 months, with the exception of the aforementioned three months of upgrading (Figure 1). Another influence on the private MRI waiting list for the QEII was found. In the fall of 2002, the IWK, a children's hospital, started allocating some of their MRI scanner time to adult cases. Because of these confounding factors, we were not able to attribute the decreasing trend in waiting lists solely to the introduction of the private MRI clinic. 
A.

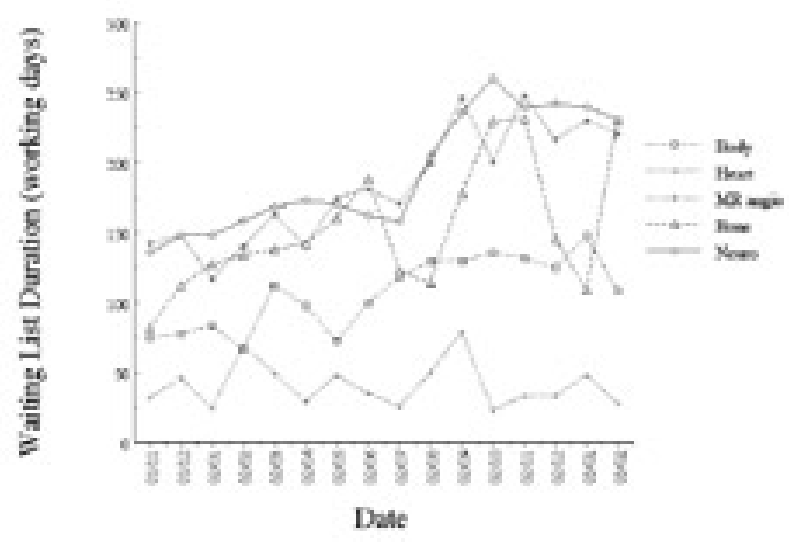

B.

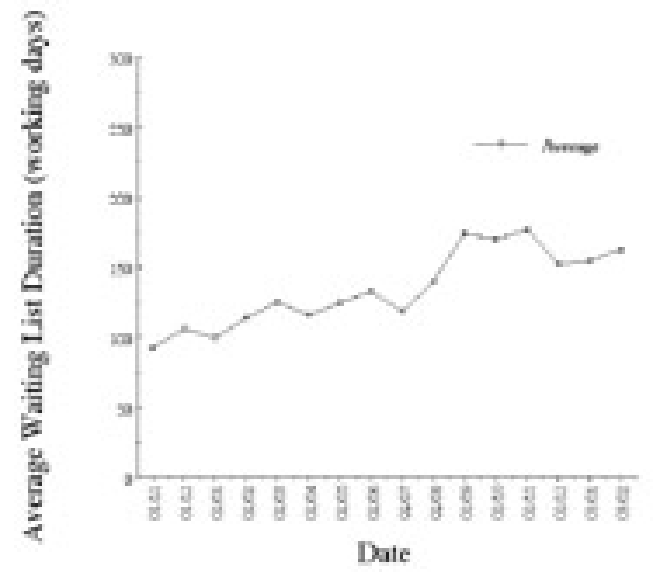

Figure 2. Waiting times for elective MRI procedures. A, Waiting times for specific elective procedures versus time. $B$, Average wait time for any elective procedure versus time.

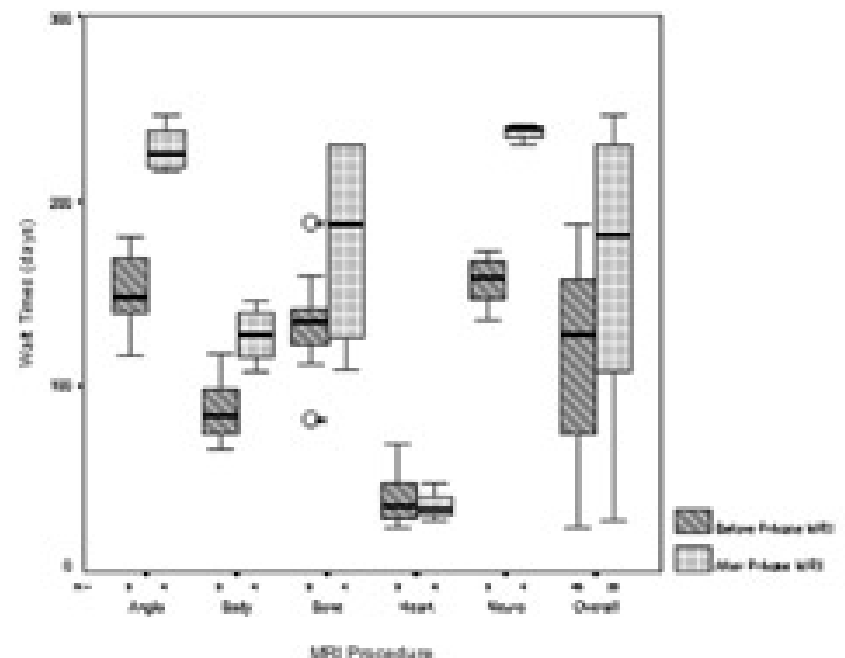

Figure 3. Wait times before and after introduction of private MRI

\section{Discussion}

Waiting lists can be evaluated, in part, with the economic concepts of supply and demand. Waiting lists will occur, in theory, when demand exceeds supply. Supply of service is dependent not only on the dominant capital investment for equipment ${ }^{11}$, but also on ongoing costs. ${ }^{12}$ Some of these costs may be reduced with economies of scale (large bulk acquisitions that are cheaper than smaller individual purchases) and savings can arise from resource sharing, such as staff pooling when multiple scanners are in close geographic proximity. ${ }^{13}$ The manner in which scans are performed and ordered have a great influence on patient throughput and thus supply of service. $^{14,15}$

The microeconomic analysis of MRI supply and demand may deviate from basic economic principles. An increase in funding (supply) might not necessarily lead to a direct reduction in waiting lists. It has been noted that with reduced waiting lists, there may be an increase in demand. ${ }^{1,13}$ Clinical decision-making necessitates consideration of given resources and access to those resources. By increasing resources
Table 1: Comparison of waiting lists before and after opening of private MRI clinic

\begin{tabular}{llll}
\hline Procedure & DF & $\mathbf{t}$ & $\mathbf{P}$ \\
\hline Overall & 63 & 2.8985 & 0.003 \\
Body & 11 & 3.6296 & 0.002 \\
Heart & 11 & 0.5404 & 0.700 \\
Angiography & 11 & 6.6042 & $<0.001$ \\
Bone & 11 & 1.8174 & 0.048 \\
Neuro & 11 & 12.5388 & $<0.001$ \\
\hline
\end{tabular}

with an MRI machine, for example, clinical decisions may be improved in the treatment of some patients. Pathology viewed by CT scanner might be better evaluated with MRI. The introduction of new MRI scanners might conceivably increase the total number of scans in a given region and possibly increase wait times.

Demand has been increasing since the introduction of MRI to the diagnostic imaging mainstream. New protocols for MRI imaging that reduce certain surgical waiting lists and relatively new procedures such as magnetic resonance angiography make MRI a coveted diagnostic tool. ${ }^{16-18}$ As evidence, the Centre for Health Services Studies of the University of Warwick suggests the demand for MRI technology will grow between 12.5 and 18.5 percent per annum based on research with National Health Service Trusts, MRI suppliers and radiologists in the United Kingdom. ${ }^{13}$

Survey respondents from the McDonald et al. initiative suggested that inadequate funding, an ageing population, and poor management of current resources were the major causes of increased waiting lists. ${ }^{1}$ Most advocated enhanced funding and improved co-ordination of resources as principal remedies. The authors suggested the following:

- Standardised methods for measuring and reporting waiting times should be developed.

- Standards need to be in place for the assessment of clinical severity including potential benefit and risk deterioration with treatment and standard methods of assigning patients to wait lists should be developed accordingly.

- There should be accountability in the system, which might include periodic audits. 

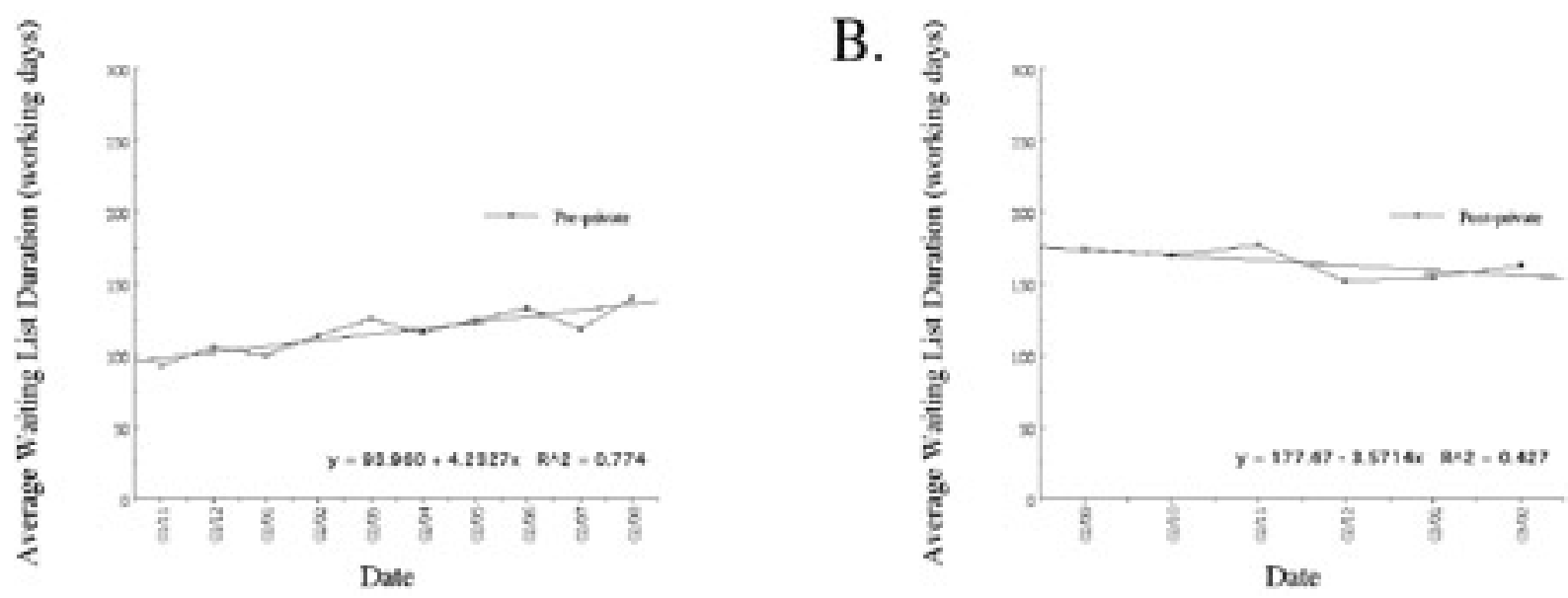

Figure 4. Trend in waiting times prior to and following the opening of the private MRI clinic in Halifax. A, Waiting times for MRI at QEII prior to the opening of the private MRI clinic are plotted versus time and trended with regression. B, Waiting times for MRI at QEII following the opening of the private MRI clinic are plotted versus time and trended with regression.

- Regional, cross-provincial, or even national registries for wait lists should be developed and maintained.

- Significant investment in wait list infrastructure needs to be put in place.

- Training of individuals skilled at critical appraisal and implementation should be funded.

The Romanow report ${ }^{7}$ suggested:

- Procedures for managing wait lists need to be handled in a more centralised manner. These may be regional, provincial, or inter-provincial. Romanow suggests this would be best managed at regional health authorities or the provincial level.

- Duration on wait lists should be based solely on seriousness of health need.

- Training of health professionals to assess patient's needs according to set criteria needs to be put in place.

- Patients should be provided with full disclosure, risks, benefits, and alternatives to waiting. They should also be told of their perceived need, possible wait time and possible factors that might lengthen that time.

\section{Conclusion}

These suggestions have been brought forward from the national level; however, we believe they could also apply to our local situation in the Halifax region. It is clear that the logistical aspects of dealing with wait lists need further evaluation.

We have found no evidence that the private MRI clinic makes a significant impact on waiting times for public MRI. The medical community must therefore focus attention on the management of public wait lists and develop more standardisation for MRI imaging. Government should not only look at capital acquisition and equipment upkeep costs for MRI technology, but also investment in the many logistical areas surrounding the delivery of diagnostic imaging to the public.

\section{References}

1. McDonald P, Shortt S, Sanmartin C, Barer M, Lewis S, Sheps $\mathrm{S}$. Waiting lists and waiting times for health care in Canada. More management!! More money?? Ottawa: Health Canada. 1998. <http://www.hc-sc.gc.ca/english/ media/releases/waiting_list.html $>$

2. Noseworthy TW, McGurran JJ, Hadorn DC. Waiting for scheduled services in Canada: development of priority-setting scoring systems. J Eval Clin Pract 2003; 9:23-31.

3. Hadorn DC. Developing priority criteria for magnetic resonance imaging: results from the Western Canada Waiting List Project. Can Assoc Radiol J 2002; 53:210-8.

4. Lewis S, Barer ML, Sanmartin C, Sheps S, Shortt SE, McDonald PW. Ending waiting-list mismanagement: principles and practice. CMAJ 2000; 162:1297-300.

5. Bell CM, Crystal M, Detsky AS, Redelmeier DA. Shopping around for hospital services: a comparison of the United States and Canada. JAMA 1998; 279:1015-7.

6. "Nova Scotia doctors want minimum standards at private MRI clinics." CBC NEWS. 30 Dec $2002<$ www.cbc. $\mathrm{ca} /$ story/canada/national/2002/12/30/mri021230.html> (20 Jan 2005).

7. Romanow RJ. "Building on values: the future of health care in Canada. Saskatoon: Commission on the Future of Health Care in Canada." 2002. <http://www.hc-sc.gc. ca/english/care/romanow/hcc0086.html> (20 Jan 2005).

8. Canadian Association of Radiologists. $2003<\mathrm{http}$ ://www. car.ca/politics/romanow/Romanow_Essential.pdf $>$ (20 Jan 2005).

9. "Report renews debate over private MRI clinics." CTV News. 28 Nov 2002. <http://www.ctv.ca/servlet/ArticleNews/story/CTVNews/1038532378035_69 > (20 Jan 2005).

10. "N.S. government says it can't block private MRI clinic." CBC News. 24 Jul $2002<$ www.cbc.ca/story/canada/national/2002/07/23/mri_ns020723.html > (20 Jan 2005). 
11. Szczepura AK, Fletcher J, Fitz-Patrick JD. Cost-effectiveness of magnetic resonance imaging. BMJ 1992; 304:183-4.

12. Fletcher J, Clark MD, Sutton FA, Wellings R, Garas K. The cost of MRI: changes in costs 1989-1996. Br J Radiol $1999 ; 72: 432-7$.

13. Szczepura A, Clark M. Creating a strategic management plan for magnetic resonance imaging (MRI) provision. Health Policy 2000; 53:91-104.

14. Moore NR, Golding SJ. Increasing patient throughput in magnetic resonance imaging: a practical approach. Oxford MRI Group. Br J Radiol 1992; 65:470-5.

15. Chawda SJ, Watura R, Lloyd DC. Magnetic resonance imaging of the lumbar spine: direct access for general practioners. Br J Gen Pract 1997; 47:575-6.

16. Swan JS, Fryback DG, Lawrence WF, Saintfort F, Hagenauer ME, Heisey DM. A time-tradeoff method for cost-effectiveness models applied to radiology. Med Decis Making 2000; 20:79-88.

17. Warwick DJ, Cavanagh P, Bell M, Marsh CH. Influence of magnetic resonance imaging on a knee arthroscopy waiting list. Injury 1993; 24:380-2.

18. Williams RL, Williams LA, Watura R, Fairclough JA. Impact of MRI on a knee arthroscopy waiting list. Ann R Coll Surg Engl 1996; 78:450-2.
COME CARE WITH US IN CAPE BRETON ... ANESTHESIOLOGIST a career challenge... a lifestyle choice

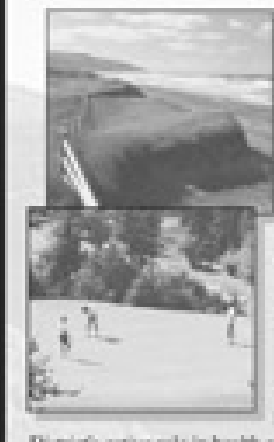

ENJOY THE CHALEVGE

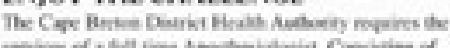

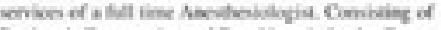

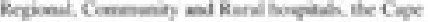

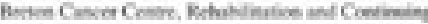

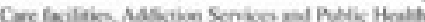

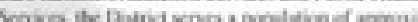

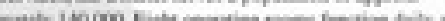

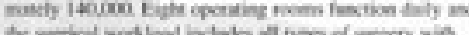

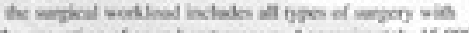

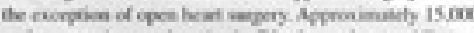

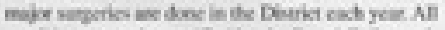

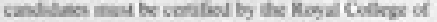
Mysiciom ind Sergecen of Cinale and ke clighte for Sonsuere in Nona Soncia

\section{EJOY THE UISTYU}

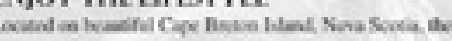

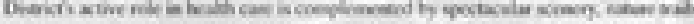

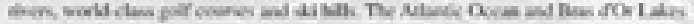

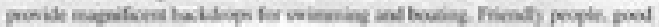

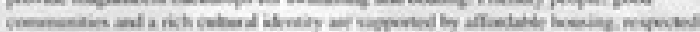

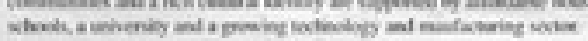

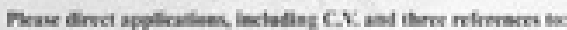

De M. A. Nopuc Medical Berector

Cope Bretes bederke Heshls Aeilerity

1482 Goowe Senel Sydery

Nowa Soda Bir IP]

Pas: (rover 569.7921

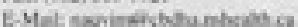

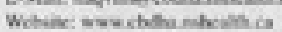

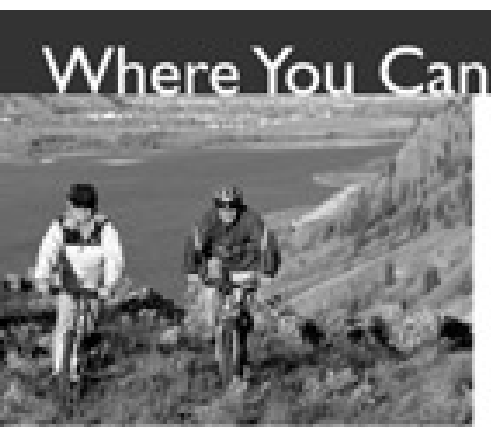

The southern interior of British

Columbia is a fourseason

recreational playground where

you can work - and play - all in

the same day, Ski backcountry

or powder, paddle pristine lakes,

or hike and bike endless trails.

Whether you locate in the sunny

Okanagan, Thompson-Cariboo-

Shuswap or Kootenays, these are

dream locations where homes are

affordable, schools are excellent

and life here is simply...easier.
Interior Health, is one of stix health wahorities

in $B C$. With an annual budget of $\$ 1,1$ bilion.

Interior Health provides a full spectrum of health care services to a population of 690.000 .

Our region is growing with immediate opportunities in family practice and a wide range of medical and surgical specialties.

Physicians within Ineerior Health receive scme of the highent levels of remuneration for health care professionals in Canada and many qualify for BC's new and highly competitive on-call payment program. Physicians in most of our smaller communities also qualify for Rural Recruitment and Retention incentives.

\section{Interior Health wwwinteriorthealth.cal}

In paresership wish health muxch bx wwwhealthmatchbc.org
Call us = then come visit - and dicover why living and working in leterior Health is a dream job in a dream location.

For more information, please contact Dr. Seeve Hardwicke, Senior Medical Director, Interior Health

2180 Ethel Street, Kelowna, BC VIY 3AI Tel 250-861-4264 Fax 250-862-4201

Email: drsteve.hardwicke (9) interiorhealth.ca

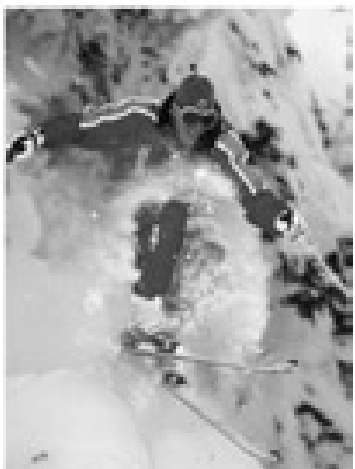

